\title{
Piecewise Constant Histogram Specification for False Contour-Free Contrast Enhancement
}

\author{
Hengjun Yu, Kohei Inoue*, Kenji Hara, and Kiichi Urahama \\ Kyushu University, 4-9-1, Shiobaru, Minami-ku, Fukuoka 815-8540, Japan \\ *Corresponding Author: k-inoue@design.kyushu-u.ac.jp
}

\begin{abstract}
We propose an algorithm for enhancing the contrast of grayscale images by piecewise constant histogram specification, which produces a contrast-enhanced image, the histogram of which is piecewise constant, and has no gap between neighboring intensities. As a result, false contouring caused by the intensity gap can be avoided. In order to evaluate the performance of contrast enhancement methods, we incorporate two conventional image quality measures into a single one. Experimental results show that the proposed algorithm outperforms two conventional histogram equalization methods in terms of the image quality measure.
\end{abstract}

Keywords: contrast enhancement, histogram specification, histogram equalization, false contour.

\section{Introduction}

Histogram specification (HS) or histogram matching, which includes histogram equalization (HE) as a special case, is a technique for intensity transformation ${ }^{(1)}$, the main purpose of which is contrast enhancement (CE). Conventional $\mathrm{HE}^{(1)}$ is a useful tool for $\mathrm{CE}$ because the target histogram is a constant function which has no parameter to be set. However, for digital images, $\mathrm{HE}^{(1)}$ cannot equalize the resultant histogram in a strict sense, and tends to overenhance the contrast of low contrast images to cause false contouring.

Coltuc et al. ${ }^{(2)}$ proposed an exact HS technique based on the strict ordering on image pixels. Once ordering is achieved, pixels are immediately separated into classes and assigned to the desired gray level. Nikolova and Steidl pointed out the importance of a meaningful strict ordering of pixels for HS, and proposed a fast ordering algorithm ${ }^{(3)}$. They also applied their algorithm to hue and range preserving color image enhancement ${ }^{(4)}$.

In general, HS accepts arbitrary shapes of target histograms including equalized (uniform), Gaussian and exponential histograms. However, specifying a meaningful histogram for a certain image is not obvious ${ }^{(2)}$. For example, a Gaussian histogram used in Ref. (4) has two parameters to be given by users. To overcome such difficulties in HS, we propose a method for generating a target histogram from a given grayscale image only. The generated histogram is piecewise constant, and has no gap between neighboring intensities. Therefore, the proposed algorithm can suppress false contouring and contrast over-enhancement which are frequently observed in contrast-enhanced images by conventional HEs.

Celik ${ }^{(5)}$ quantitatively evaluated the performance of contrast enhancement algorithms by using two measures, i.e., the expected measure of enhancement by gradient (EMEG) and the gradient magnitude similarity deviation (GMSD) proposed by Xue et al. ${ }^{(6)}$. EMEG ${ }^{(5)}$ is a modification of EME by entropy (EMEE) proposed by Agaian et al. ${ }^{(7)}$. In this paper, we incorporate $\mathrm{EMEG}^{(5)}$ and $\mathrm{GMSD}^{(6)}$ into a single measure, and evaluate the performance of contrast enhancement algorithms using the measure. Experimental results show that the proposed algorithm achieves higher (better) values of the measure than conventional $\mathrm{HEs}^{(1),(3),(4)}$.

\section{Piecewise Constant Histogram Specification}

In this section, we describe the proposed piecewise constant histogram specification algorithm, which fills 0valued bins of the histogram equalized by Conventional $\mathrm{HE}^{(1)}$ with non-zero values of neighboring bins. The repeated copies of non-zero values to the neighboring consecutive 0 valued bins generate a piecewise constant histogram, with 
which the histogram-specified image is finally obtained.

Let $F=\left[f_{i j}\right]$ be a grayscale image of size $m \times n$ pixels, where $f_{i j} \in\{0,1, \ldots, L-1\}$ denotes the intensity at the position $(i, j)$ for $i=1,2, \ldots, m$ and $j=1,2, \ldots, n$, where $L$ denotes the number of intensity levels, e.g., $L=$ $2^{8}=256$ for 8-bit images. Let $\boldsymbol{h}^{F}=\left[h_{0}^{F}, h_{1}^{F}, \ldots, h_{L-1}^{F}\right]$ be the histogram of $F$, where $h_{k}^{F}=\sum_{i=1}^{m} \sum_{j=1}^{n} \delta_{f_{i j}, k} \quad$ for $k=$ $0,1, \ldots, L-1$, where $\delta_{f_{i j}, k}$ denotes the Kronecker delta. Let $\boldsymbol{H}^{F}=\left[H_{0}^{F}, H_{1}^{F}, \ldots, H_{L-1}^{F}\right]$ be the cumulative histogram of $\boldsymbol{h}^{F}$, where $H_{k}^{F}=\sum_{k^{\prime}=0}^{k} h_{k^{\prime}}^{F}$. Then the histogram-equalized image $G=\left[g_{i j}\right]$ of $F$ is given by $g_{i j}=(L-1)\left(H_{f_{i j}}^{F}-\right.$ $\left.H_{\min }^{F}\right) /\left(H_{\max }^{F}-H_{\min }^{F}\right)$, where $H_{\max }^{F}=H_{f_{\max }}^{F}=m n$ and $H_{\text {min }}^{F}=H_{f_{\min }}^{F}$, where $f_{\max }=\max \left\{f_{i j}\right\}$ and $f_{\min }=\min \left\{f_{i j}\right\}$.

For a low contrast image $F$, the histogram $\boldsymbol{h}^{G}$ of the histogram-equalized image $G$ frequently becomes sparse, i.e., a number of elements of $\boldsymbol{h}^{G}$ are zero. The sparsity of $\boldsymbol{h}^{G}$ indicates that there exist gaps in continuous-tone, which may cause false contours in $G$. To bridge those gaps, we fill all bins of $\boldsymbol{h}^{G}$ with non-zero values as follows: Let $\widetilde{\boldsymbol{h}}^{G}=$ $\left[\tilde{h}_{k}^{G}\right]$ be the filled histogram, which is initialized as $\widetilde{\boldsymbol{h}}^{G}:=$ $\boldsymbol{h}^{G}$. Let $\bar{f}=\sum_{k=0}^{L-1} k h_{k}^{F} / \sum_{k=0}^{L-1} h_{k}^{F}$ and $\bar{g}=\sum_{k=0}^{L-1} k h_{k}^{G} /$ $\sum_{k=0}^{L-1} h_{k}^{G}$ be the mean intensities of $F$ and $G$, respectively. If $\bar{g}<\bar{f}$, then, for $k=1,2, \ldots, L-1$ in ascending order, we fill $\tilde{h}_{k}^{G}=0$ with its neighbor on the left as $\tilde{h}_{k}^{G}:=$ $\tilde{h}_{k-1}^{G} \neq 0$. On the other hand, if $\bar{g} \geq \bar{f}$, then, for $k=L-$ $2, L-3, \ldots, 0$ in descending order, we fill $\tilde{h}_{k}^{G}=0$ with its neighbor on the right as $\tilde{h}_{k}^{G}:=\tilde{h}_{k+1}^{G} \neq 0$. Next, we scale $\widetilde{\boldsymbol{h}}^{G}$ so that the total sum of the elements coincides with $m n$, i.e., $\widehat{\boldsymbol{h}}^{G}:=m n \widetilde{\boldsymbol{h}}^{G} / \sum_{k=0}^{L-1} \tilde{h}_{k}^{G}$. As a result, we obtain the scaled and filled histogram $\widehat{\boldsymbol{h}}^{G}$ of which every bin has non-zero value.

Next, we transform $F$ into another image $\hat{G}=\left[\hat{g}_{i j}\right]$ the histogram of which coincides with $\widehat{\boldsymbol{h}}^{G}$. This procedure is an example of histogram specification ${ }^{(2)}$. We adopt the stateof-the-art technique proposed by Nikolova and Steidl ${ }^{(3),(4)}$.

The proposed algorithm is summarized as follows:

\begin{tabular}{l} 
Algorithm 1 Piecewise Constant Histogram Specification \\
\hline Require: original grayscale image $F$ \\
Ensure: contrast-enhanced image $\widehat{G}$ \\
1: Compute the histogram $\boldsymbol{h}^{F}$ of $F$; \\
2: Compute the cumulative histogram $\boldsymbol{H}^{F}$ of $\boldsymbol{h}^{F}$; \\
3: Compute the histogram-equalized image $G$ of $F$; \\
4: Compute the histogram $\boldsymbol{h}^{G}$ of $G$; \\
5: Compute the mean intensities $\bar{f}$ and $\bar{g}$ of $F$ and $G$;
\end{tabular}

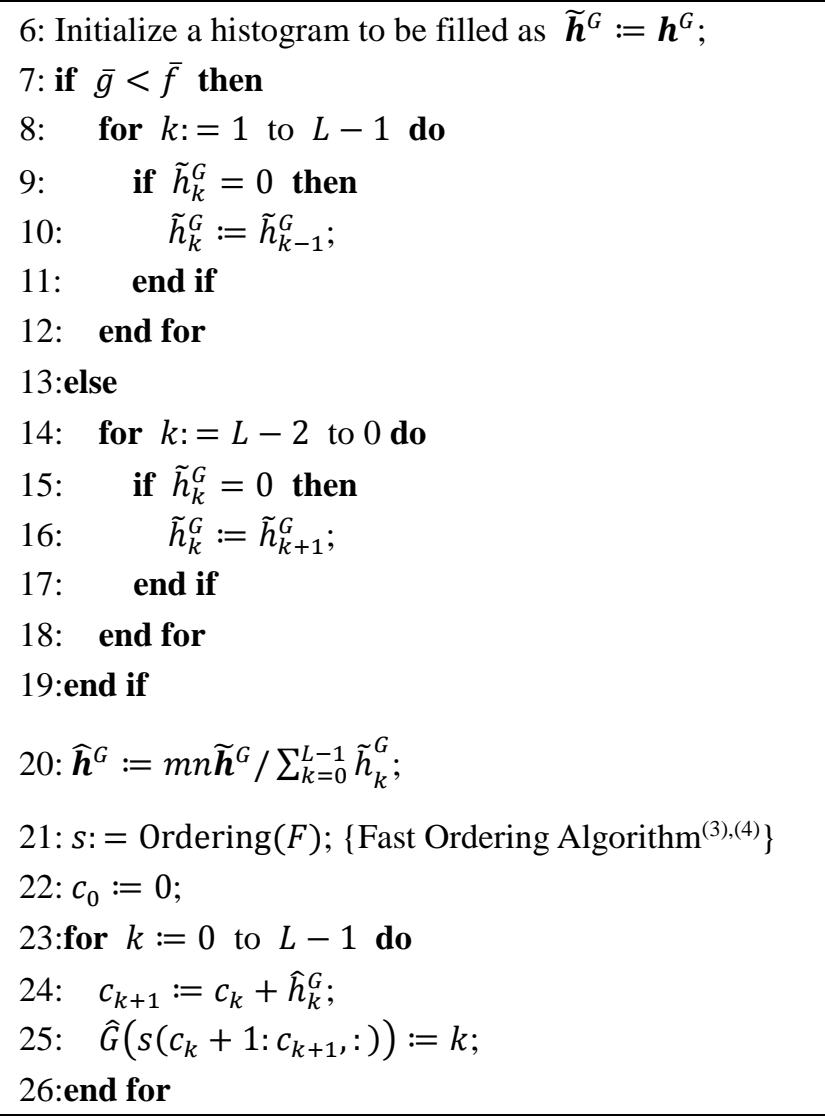

In Algorithm 1, line 21, the function Ordering returns an $m n \times 2$ array $s$ in which the positions $(i, j)$ of pixels are stored in ascending order of the pixel values obtained by the fast ordering algorithm ${ }^{(3),(4)}$. Each intensity $k \in$ $\{0,1, \ldots, L-1\}$ is assigned to the corresponding pixels in line 25, where we use the colon notations in MATLAB.

In summary, Algorithm 1 fills the empty bins in equalized histograms by copying the neighboring non-zero elements, and preserves the outline of original histograms.

\section{Image Quality Measures}

In this section, we briefly summarize two measures for evaluating the performance of contrast enhancement methods, and then incorporate them into a single measure.

\subsection{Expected measure of enhancement by gradient ${ }^{(5)}$}

The expected measure of enhancement by gradient (EMEG) proposed by Celik ${ }^{(5)}$ is defined by

$$
\operatorname{EMEG}(F)=\frac{1}{M N} \sum_{I=1}^{M} \sum_{J=1}^{N} \frac{1}{\beta} \max \left(\frac{F_{I J}^{d x, h}}{F_{I, J}^{d x, l}+\epsilon}, \frac{F_{I, J}^{d y, h}}{F_{I, J}^{d y, l}+\epsilon}\right),
$$

where $F_{I, J}^{d x, h}\left(F_{I, J}^{d y, h}\right)$ and $F_{I, J}^{d x, l}\left(F_{I, J}^{d y, l}\right)$ are the maximum and minimum values of absolute valued derivatives in horizontal (vertical) direction of the $w_{1} \times w_{2}$ sub-block 
$F_{I, J}$ of an image $F, \beta=L-1$, and $\epsilon$ is a positive constant. We set $\epsilon=1, w_{1}=w_{2}=8, M=\operatorname{fix}\left(m / w_{1}\right)$ and $N=\operatorname{fix}\left(n / w_{2}\right)$ where fix denotes a function for rounding toward zero. When $G$ is a contrast-enhanced image of $F$, we expect that $\operatorname{EMEG}(G)>\operatorname{EMEG}(F)$.

\subsection{Gradient magnitude similarity deviation ${ }^{(6)}$}

The gradient magnitude similarity deviation (GMSD) proposed by Xue et al. ${ }^{(6)}$ is a full reference image quality assessment model defined by

$$
\operatorname{GMSD}(F, G)=\sqrt{\frac{1}{m n} \sum_{i=1}^{m} \sum_{j=1}^{n}\left(\mathrm{GMS}_{i j}^{F, G}-\operatorname{GMSM}^{F, G}\right)^{2}},
$$

where $\mathrm{GMSM}^{F, G}$ denotes the average of $\mathrm{GMS}_{i j}^{F, G}$ which is the value of the GMS map at $(i, j)$ defined by

$$
\mathrm{GMS}_{i j}^{F, G}=\frac{2 m_{F, i j} m_{G, i j}+c}{m_{F, i j}^{2} m_{G, i j}^{2}+c},
$$

where $m_{F, i j}$ and $m_{G, i j}$ denote the gradient magnitudes of $F$ and $G$ at $(i, j)$, respectively, and $c$ is a positive constant for numerical stability. A lower value of GMSD means higher quality. The MATLAB source code for calculating GMSD can be downloaded at http://www4.comp.polyu.edu.hk/ cslzhang/IQA/GMSD/G MSD.htm. We used it with the default settings.

\subsection{Incorporating EMEG ${ }^{(5)}$ and GMSD ${ }^{(6)}$}

In this subsection, we propose an image quality measure which incorporates EMEG ${ }^{(5)}$ and $\mathrm{GMSD}^{(6)}$ into a single value which we call EMEG over GMSD (EMEG/GMSD), which is defined by

$$
\operatorname{EMEG} / \operatorname{GMSD}(F, G)=\frac{\operatorname{EMEG}(F)}{\operatorname{GMSD}(F, G)} \text {. }
$$

The higher the EMEG/GMSD value is, the higher the performance of contrast enhancement is. This property is consistent with that of both $\mathrm{EMEG}^{(5)}$ and $\mathrm{GMSD}^{(6)}$.

\section{Experimental Results}

We compared the proposed algorithm with the conventional HE ${ }^{(1)}$ and Nikolova and Steidl's one ${ }^{(3),(4)}$ using the SIDBA standard image database ${ }^{(8)}$. Fig. 1 shows an original image and the contrast-enhanced ones in the left column and the corresponding histograms in the right. The original image in Fig. 1(a) has the histogram in Fig. 1(b), which provides a graphical evidence that the image is dark and low-contrast. The conventional HE produces a contrast-

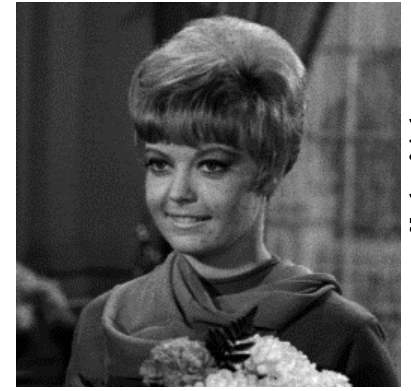

(a) Original image (Girl)

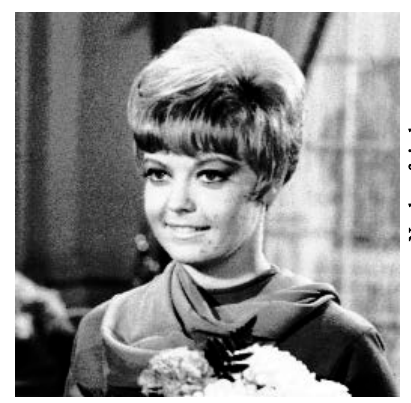

(c) Conventional $\mathrm{HE}^{(1)}$

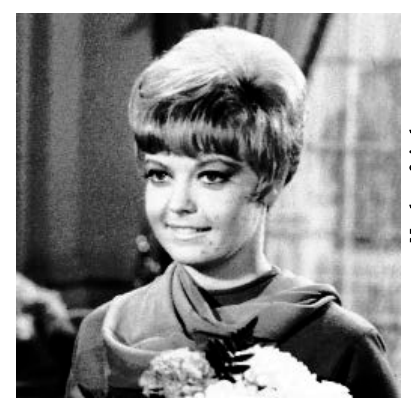

(e) Nikolova(3),(4)

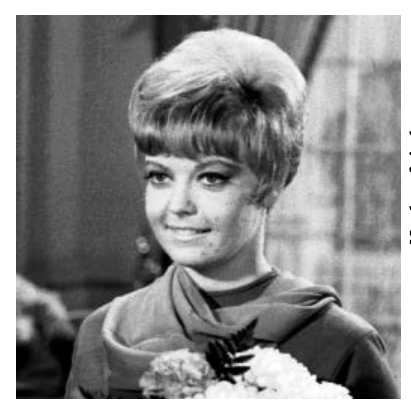

(g) Proposed

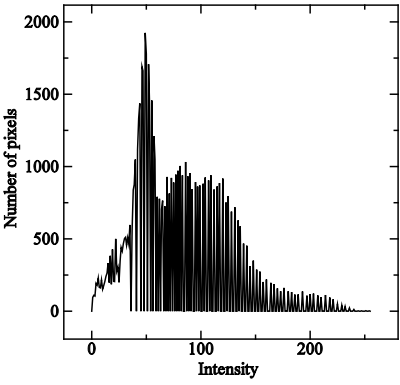

(b) Histogram of (a)

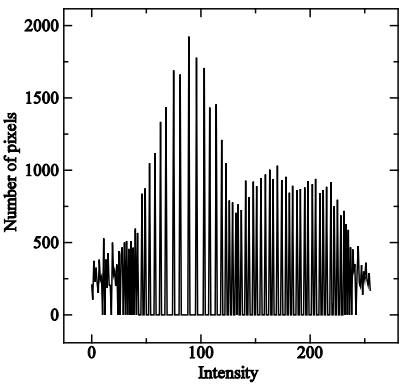

(d) Histogram of (c)

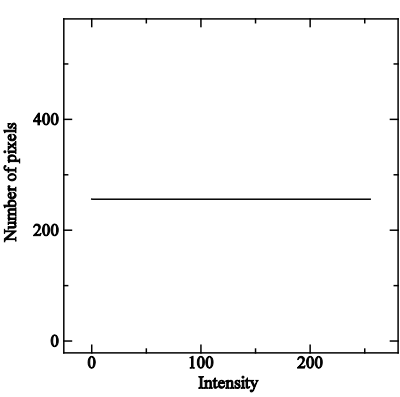

(f) Histogram of (e)

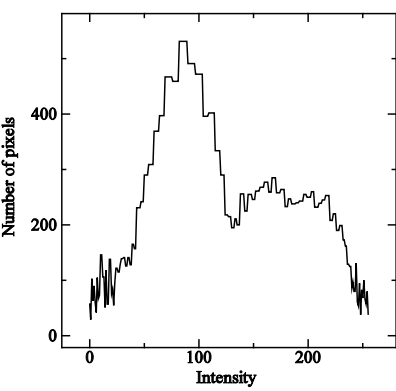

(h) Histogram of (g)
Fig. 1. Grayscale images (left) and their histograms.

Table 1. CPU time [sec.].

\begin{tabular}{|c|c|c|}
\hline Conventional & Nikolova & Proposed \\
\hline 3.08 & 24.96 & 24.98 \\
\hline
\end{tabular}

enhanced image in Fig. 1(c), the histogram of which has a comb-shaped graph as shown in Fig. 1(d). On the other hand, Nikolova's $\mathrm{HE}^{(3),(4)}$ outputs a similar image in Fig. 1(e) to that in Fig. 1(c), however, the histogram of Fig. 1(e) is exactly uniform as shown in Fig. 1(f) which is greatly different from 


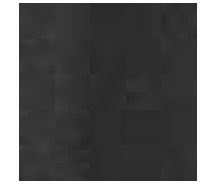

(a) Fig. 1(a)

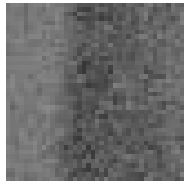

(b) Fig. 1(c)

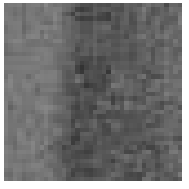

(c) Fig. 1(e)

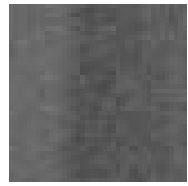

(d) Fig. 1(g)
Fig. 2. Zoomed parts.

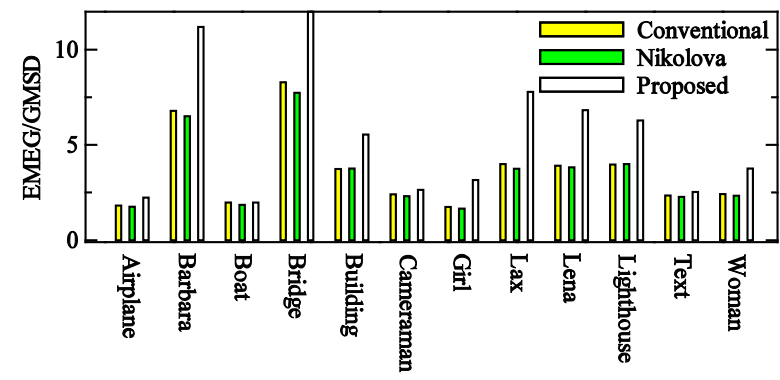

Fig. 3. EMEG/GMSD values.

Fig. 1(d). Fig. 1(g) shows the image generated by the proposed algorithm, which enhances the contrast more softly than the above HE algorithms (Figs. 1(c) and (e)). The histogram of Fig. 1(g) is shown in Fig. 1(h), which demonstrates that the proposed algorithm generates a piecewise constant histogram. Table 1 shows CPU time for computing Figs. (c), (e) and (g). The proposed algorithm requires almost the same CPU time as Nikolova's $\mathrm{HE}^{(3),(4)}$. Both the proposed algorithm and Nikolova's $\mathrm{HE}^{(3),(4)}$ spend most of the CPU time for computing the ordering of the pixel values as in Algorithm 1, line 21. We performed the experiments using MATLAB R2011b on a Pentium IV 3.4GHz machine with 2GB RAM.

We zoomed up a region of wall behind the girl as shown in Fig. 2, where Figs. 2(a)-(d) correspond to Figs. 1(a), (c), (e) and (g), respectively. Although the contrast around the belt-shaped slightly bright region is enhanced by the conventional and Nikolova's HEs, random noise is also enhanced by false contouring as shown in Figs. 2(b) and (c). On the other hand, in Fig. 2(d), the noise enhancement caused by false contouring is suppressed by the proposed algorithm.

Fig. 3 shows EMEG/GMSD values for 12 images in SIDBA $^{(8)}$. The vertical axis denotes the EMEG/GMSD value, and the horizontal axis denotes the name of each image. The yellow, green and white bars denote the conventional $\mathrm{HE}^{(1)}$, Nikolova's one ${ }^{(3),(4)}$ and the proposed algorithm, respectively. The proposed algorithm achieved higher EMEG/GMSD values than the conventional $\mathrm{HE}^{(1)}$ and Nikolova's one ${ }^{(3),(4)}$ for all 12 images.

Other contrast enhancement results are shown in Fig. 4,
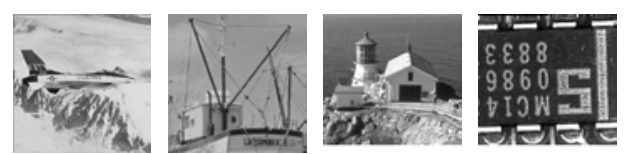

Original images
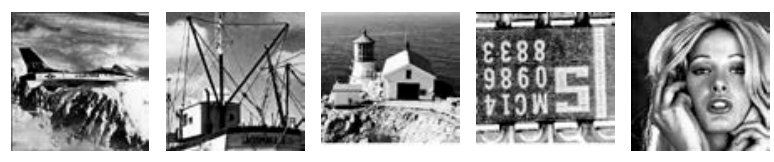

Conventional $\mathrm{HE}^{(1)}$
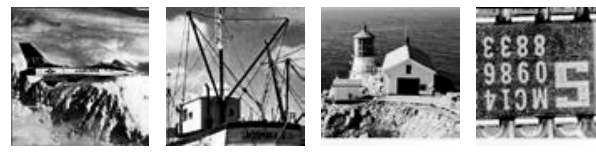

Nikolova ${ }^{(3),(4)}$
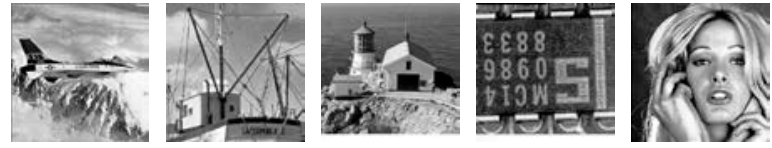

Proposed

Fig. 4. Contrast enhancement results.

where the top row shows 5 original images: Airplane, Boat, Lighthouse, Text and Woman, and the second to fourth rows show the contrast-enhanced images by the conventional $\mathrm{HE}^{(1)}$, Nikolova's one ${ }^{(3),(4)}$ and the proposed algorithm, respectively. The conventional $\mathrm{HE}^{(1)}$ and Nikolova's one $\mathrm{e}^{(3),(4)}$ tends to over-enhance the global contrast in each image, while the proposed algorithm can alleviate the contrast overenhancement to obtain appropriate results.

\section{Conclusions}

In this paper, we proposed a contrast enhancement algorithm, by which the contrast-enhanced images have piecewise constant histograms. As a result, the gaps of intensity values, which may cause false contours, is bridged, and the proposed algorithm outputs proper results of contrast enhancement compared with conventional histogram equalization methods. The performance of the proposed algorithm is evaluated quantitatively and objectively by an image quality measure.

\section{References}

(1) R. C. Gonzalez and R. E. Woods : Digital Image Processing, Pearson Prentice Hall, 3rd ed., 2008

(2) D. Coltuc, P. Bolon, and J.-M. Chassery: "Exact Histogram Specification”, IEEE Trans. Image Process., Vol. 15, No. 5, pp. 1143-1151, 2006

(3) M. Nikolova and G. Steidl : "Fast Ordering Algorithm for Exact histogram Specification”, IEEE Trans. Image Process., Vol. 23, No. 12, pp. 5274-5283, 2014 
(4) M. Nikolova and G. Steidl : "Fast Hue and Range Preserving Histogram Specification: Theory and New Algorithms for Color Image Enhancement”, IEEE Trans. Image Process., Vol. 23, No. 9, pp. 4087-4100, 2014

(5) T. Celik : "Spatial Entropy-Based Global and Local Image Contrast Enhancement”, IEEE Trans. Image Process., Vol. 23, No. 12, pp. 5298-5308, 2014

(6) W. Xue, L. Zhang, X. Mou, and A. C. Bovik : "Gradient Magnitude Similarity Deviation: A Highly Efficient Perceptual Image Quality Index”, IEEE Trans. Image Process., Vol. 23, No. 2, pp. 684-695, 2014

(7) S. S. Agaian, B. Silver, and K. A. Panetta : “Transform Coefficient Histogram-Based Image Enhancement Algorithms Using Contrast Entropy”, IEEE Trans. Image Process., Vol. 16, No. 3, pp. 741-758, 2007

(8) M. Sakauchi, Y. Ohsawa, M. Sone, and M. Onoe : "Management of the standard image database for image processing researches”, ITEJ Technical Report, Vol. 8, No. 38, pp. 7-12, 1984 